\title{
ORT.06 - Development of lentiviral vectors for inhibition of hepatitis B virus, via small interfering RNAi
}

Bárbara Vieira do Lago ${ }^{1 *}$; Nayhanne Tizzo de Paula ${ }^{1}$; Giovana Angelice ${ }^{2}$; Francisco C. A. Mello $^{2}$; Vanessa de Paula ${ }^{2}$; Maryrose Lavatori²; Francisco José Aragão ${ }^{3}$; Elisabeth Lampe ${ }^{2}$.

1Fiocruz/Bio-Manguinhos;

2Fiocruz/IOC;

3Embrapa Pecuária Sul.

Introduction: It is estimated that chronic hepatitis B virus (HBV) infection accounts for approximately one million deaths per year due to severe cirrhosis and hepatocellular carcinoma (HCC). Currently, several drugs are used in the treatment of chronic hepatitis B, however, complete cure is still controversial. The major challenge is the persistence of viral covalently closed circular DNA (cccDNA), as well as the ability of HBV to integrate into the host genome, which enables the infection's reactivation. Interfering RNA (RNAi) is a post-transcriptional mechanism of gene silencing and demonstrates to be a promising alternative for the treatment of chronic hepatitis $\mathrm{B}$.

Objective: The aim of this study is to obtain an effective lentiviral vector in the silencing of different HBV proteins, via RNAi.

Methodology: After DNA synthesis, the shRNA coding sequences were transferred to the expression vector (CS-RfA-EG) using the Gateway recombination technology. The efficiency of silencing by siRNA candidates, used individually or in combination, will also be assessed by quantification of HBeAg proteins by commercial immunoassay and HBV DNA by real-time PCR during the post-infection period.

Results: Three silencing vectors candidates were obtained and tested in silico in order to eliminate off-target effects. Stability and secondary structures have also been tested. Such molecules are able to deliver short hairpin molecules RNAs (shRNAs) that will generate small interfering RNAs (siRNAs) targeting overlapped Open Reading Frames (ORFs), allowing different viral proteins and the pre-genomic RNA to be silenced with a single RNAi. The first lentiviral construct, targeting S/Pol genes, was obtained successfully. Huh7 cells were transfected with HBV DNA and 3 days later, infected with the first lentiviral candidate. From the third day post-infection, HBsAg became undetectable on cells treated with the construct, while untreated controls maintained viral protein expression.

Conclusion: Silencing of HBsAg was achieved from the day 3 post-infection. This approach allows long-term, sustained knockdown of HBV replication and gene expression, which can effectively eliminate HBV from chronic carriers.

Keywords: HBV; Lentiviral vectors; siRNA 